\title{
Anti-malarial efficacy and resistance monitoring of artemether-lumefantrine and dihydroartemisinin-piperaquine shows inadequate efficacy in children in Burkina Faso, 2017-2018
}

Adama Gansané ${ }^{*}$, Leah F. Moriarty² ${ }^{2}$ Didier Ménard ${ }^{3}$, Isidore Yerbanga ${ }^{4}$, Esperance Ouedraogo ${ }^{1}$, Paul Sondo ${ }^{4}$, Rene Kinda ${ }^{1}$, Casimir Tarama', Edwige Soulama' ${ }^{1}$ Madou Tapsoba', David Kangoye ${ }^{1}$, Cheick Said Compaore ${ }^{5}$, Ousmane Badolo ${ }^{6}$, Blami Dao ${ }^{6}$, Samuel Tchwenko ${ }^{2}$, Halidou Tinto ${ }^{4}$ and Innocent Valea ${ }^{4}$

\begin{abstract}
Background: The World Health Organization recommends regularly assessing the efficacy of artemisinin-based combination therapy (ACT), which is a critical tool in the fight against malaria. This study evaluated the efficacy of two artemisinin-based combinations recommended to treat uncomplicated Plasmodium falciparum malaria in Burkina Faso in three sites: Niangoloko, Nanoro, and Gourcy.

Methods: This was a two-arm randomized control trial of the efficacy of artemether-lumefantrine (AL) and dihydroartemisinin-piperaquine (DP). Children aged 6-59 months old were monitored for 42 days. The primary outcomes of the study were uncorrected and PCR-corrected efficacies to day 28 for AL and 42 for DP. Molecular markers of resistance to artemisinin derivatives and partner drugs were also analysed.

Results: Of 720 children enrolled, 672 reached study endpoints at day 28, 333 in the AL arm and 339 in the DP arm. PCR-corrected 28-day per protocol efficacy in the AL arm was 74\% (64-83\%) in Nanoro, 76\% (66-83\%) in Gourcy, and $92 \%(84-96 \%)$ in Niangoloko. The PCR-corrected 42-day per protocol efficacy in the DP arm was 84\% (75-89\%) in Gourcy, 89\% (81-94\%) in Nanoro, and 97\% (92-99\%) in Niangoloko.

No Pfk 13 mutation previously associated with artemisinin-resistance was observed. No statistically significant association was found between treatment outcome and presence of the 86Y mutation in the Pfmdr1 gene. There was also no association observed between treatment outcome and Pfpm2 or Pfmdr1 copy number variation.
\end{abstract}

Conclusion: The results of this study indicate evidence of inadequate efficacy of AL at day 28 and DP at day 42 in the same two sites. A change of first-line ACT may be warranted in Burkina Faso.

Trial Registry Pan African Clinical Trial Registry Identifier: PACTR201708002499311.

Date of registration: 8/3/2017

*Correspondence: agansane@hotmail.com

${ }^{1}$ Centre National de Recherche Et de Formation Sur Le Paludisme,

Ouagadougou, Burkina Faso

Full list of author information is available at the end of the article

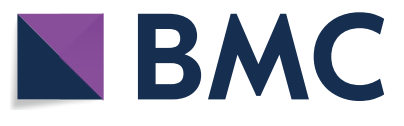

(c) The Author(s) 2021. This article is licensed under a Creative Commons Attribution 4.0 International License, which permits use, sharing, adaptation, distribution and reproduction in any medium or format, as long as you give appropriate credit to the original author(s) and the source, provide a link to the Creative Commons licence, and indicate if changes were made. The images or other third party material in this article are included in the article's Creative Commons licence, unless indicated otherwise in a credit line to the material. If material is not included in the article's Creative Commons licence and your intended use is not permitted by statutory regulation or exceeds the permitted use, you will need to obtain permission directly from the copyright holder. To view a copy of this licence, visit http://creativeco mmons.org/licenses/by/4.0/. The Creative Commons Public Domain Dedication waiver (http://creativecommons.org/publicdomain/ zero/1.0/) applies to the data made available in this article, unless otherwise stated in a credit line to the data. 
https://pactr.samrc.ac.za/Search.aspx

Keywords: Plasmodium falciparum, Artemether-lumefantrine, Dihydroartemisinin-piperaquine, Efficacy, Burkina faso, Antimalarial

\section{Background}

Despite recent encouraging global progress in malaria control, the brunt of the burden of malaria is still borne by sub-Saharan African countries [1]. In Burkina Faso, malaria is the most frequent cause of hospitalization (57\%) and death (36\%) in healthcare facilities. Plasmodium falciparum is responsible for more than $80 \%$ of malaria cases [2, 3]. In 2005, after the decline in efficacy of chloroquine [4-6], artemisinin-based combination therapy (ACT) (artemether-lumefantrine [AL] and artesunate-amodiaquine [ASAQ] $)$ was adopted as the first-line treatment for uncomplicated malaria, but only became available 2 years later [7]. Starting in 2014, the use of ASAQ at public facilities was gradually discontinued as recommended by WHO for areas where seasonal malaria chemoprevention with amodiaquine plus sulfadoxine-pyrimethamine is implemented [8]. Dihydroartemisinin-piperaquine (DP) was added as an additional first-line option in 2017 [9].

The emergence and spread of parasite resistance to artemisinin derivatives and partner drugs used in ACT jeopardizes progress in malaria control $[10,11]$. To date, mutations in the propeller domain of the Pfkelch13 gene have been associated with artemisinin resistance in Southeast Asia and identified in one country in subSaharan Africa [12, 13]. Resistance to partner drugs is also a concern; increased copy number of Plasmodium falciparum multidrug resistance 1 (Pfmdr1) and plasmepsin 2 (Pfpm2) genes represent validated molecular signatures associated with resistance to lumefantrine $[14]$ and piperaquine $[15,16]$, respectively. The World Health Organization (WHO) recommends assessing the therapeutic efficacy and safety of $\mathrm{ACT}$ at least every two years [7]. In neighbouring Mali and Niger, recent studies shown high PCR-corrected efficacy for DP (99.4\%) and varying efficacy for AL, with 28-day PCR-corrected efficacy measured at $84.5 \%$ in a recent published study in Mali [17-19].

In Burkina Faso, studies conducted between 2008 and 2012 have demonstrated PCR-corrected efficacy of AL ranging between $78-91 \%$ after 28 days follow-up [2022], However, these results were generated using alternative statistical methods to those recommended by the WHO (Table 1). This study reports data on the efficacy and the safety of AL and DP in 2017-2018 among children 6-59 months old, in addition to molecular markers associated with anti-malarial resistance, including Pfkelch13, Pfmdr1, and Pfpm2 to support evidence-based decisions on malaria treatment policy.

\section{Methods}

Study sites

The study was conducted in three sites representing differing malaria transmission zones in Burkina Faso:

- Niangoloko: The Niangoloko Medical Centre located in the Banfora Health District; malaria transmission occurs year-round with a peak during the rainy season (May-October). Malaria incidence is reported as 2349 per 1000 among children under 5 years old according to routine surveillance data [23].

- Nanoro: The Nanoro and Temnaoré primary health facilities in the Nanoro Health District; malaria transmission is stable, seasonal and occurs throughout the rainy season (May-October). Malaria incidence is reported as1979 per 1000 among children under 5 years old according to routine surveillance data [23].

- Gourcy: The Gourcy District Hospital located in the Gourcy Health District; malaria transmission is sta-

Table 1 Results of previous artemether-lumefantrine therapeutic efficacy studies recalculated in accordance with WHO recommendations

\begin{tabular}{|c|c|c|c|c|c|c|c|}
\hline \multirow[b]{2}{*}{ Year of study } & \multirow[b]{2}{*}{$\begin{array}{l}\text { Year } \\
\text { of publication }\end{array}$} & \multirow[b]{2}{*}{ Reference } & \multirow[b]{2}{*}{ Site } & \multicolumn{2}{|c|}{ PCR uncorrected 28-day efficacy } & \multicolumn{2}{|c|}{ PCR corrected 28-day efficacy } \\
\hline & & & & Published $^{\mathrm{a}}$ & Recalculated $^{\mathbf{b}}$ & Published $^{a}$ & Recalculated \\
\hline 2008-2010 & 2014 & Tinto et al. [22] & Nanoro & 46.1 & 46.1 & 89.8 & 81.9 \\
\hline 2009 & 2011 & Siribié et al. [20] & Banfora & 66.7 & 66.7 & 90.5 & 87.5 \\
\hline 2010-2012 & 2015 & Sondo et al. [21] & Nanoro & 43.3 & 43.3 & 77.8 & 64.5 \\
\hline
\end{tabular}

a Published results

b Recalculated using WHO per protocol definition [7] 
ble, seasonal and occurs throughout the rainy season (June-September); malaria incidence is reported as 1526 per 1000 in children under 5 years old according to routine surveillance data [23] (Fig. 1).

\section{Study design and randomization}

This was a phase IV randomized, open label, multi-site study assessing the efficacy and safety of AL and DP based on the WHO protocol for the surveillance of antimalarial drug efficacy [7]. Children with uncomplicated $P$. falciparum malaria who met the study inclusion criteria were screened, enrolled, assigned to treatment with AL or DP by block randomization and monitored for 42 days.

\section{Sample size estimation}

Considering a maximum acceptable treatment failure rate of $10 \%$ with a confidence level of $90 \%$ and precision of 5\%, the sample size needed was estimated at 105 patients per arm in each site; enrolment of 120 participants per arm was planned to account for withdrawal or loss to follow-up. According to the WHO, in order for the study to be representative, a minimum sample of 50 patients is required, regardless of the rates of failure.

\section{Screening and recruitment}

Children aged between 6-59 months with fever or history of fever in the previous $24 \mathrm{~h}$ seeking treatment at the targeted health facilities were examined by nurses and tested for malaria with a rapid diagnostic test (RDT) (Malaria Ag Pf/Pan, SD Bioline). Children with a positive RDT and no other illness requiring immediate attention were referred to the study team for screening procedures including clinical examination and malaria microscopy. Children were enrolled in the study if the following criteria were met: (a) mono-infection with $P$. falciparum detected by microscopy, (b) asexual parasite count of 2000-200 000/ $\mu$ l, (c) axillary temperature $\geq 37.5{ }^{\circ} \mathrm{C}$ or reported fever during the previous $24 \mathrm{~h}$, (d) haemoglobin level $\geq 5 \mathrm{~g} / \mathrm{dl}$, (e) ability to swallow oral medication, (f) ability and willingness of caregivers to comply with the protocol for the duration of the study, (g) no history of effective anti-malarial treatment in the preceding $72 \mathrm{~h}$ and (h) written informed consent of a parent or guardian. Other selection criteria were evaluated according to the WHO standardized protocol [7]. Children who did

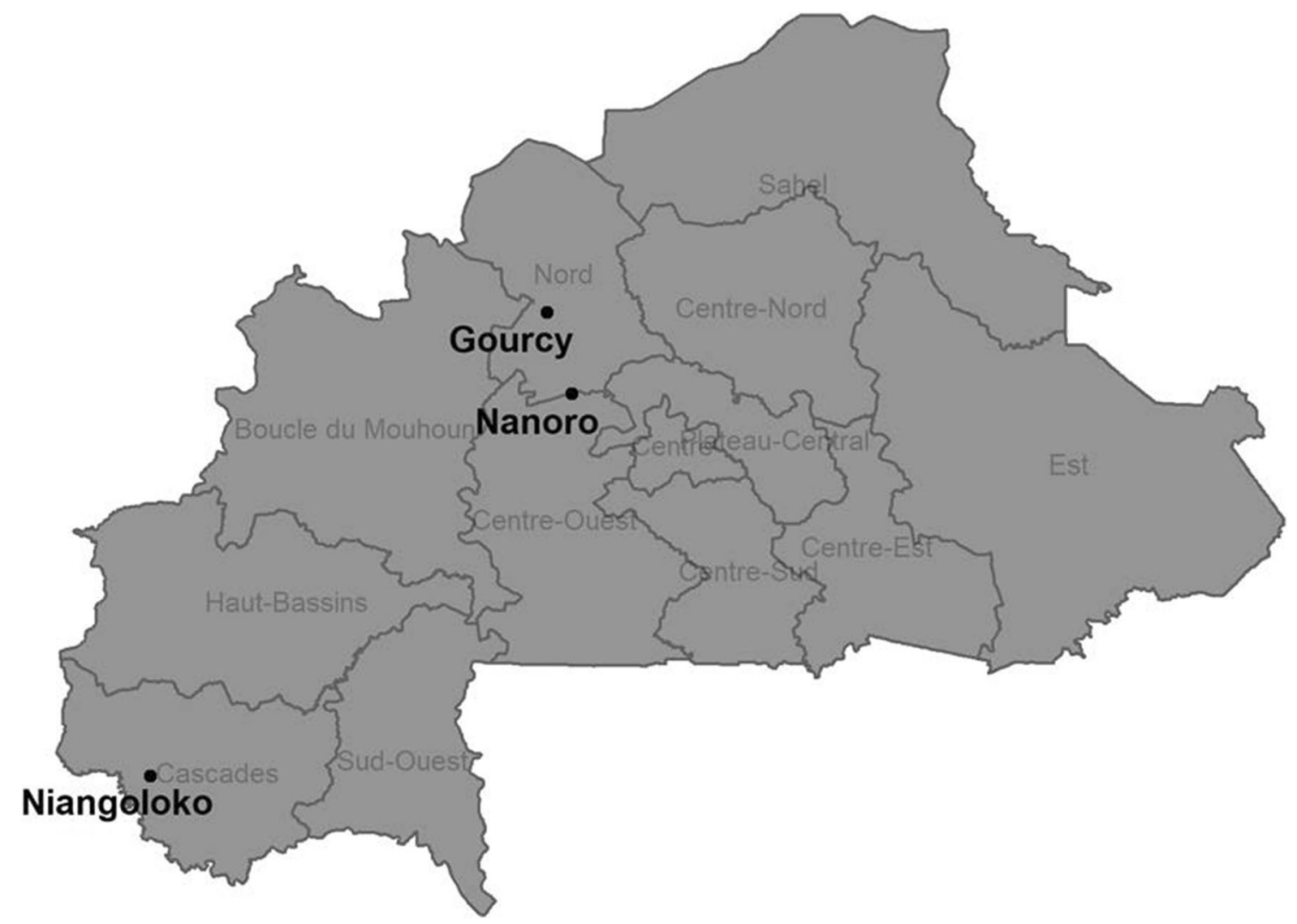

Fig. 1 Map of therapeutic efficacy study sites, Burkina Faso, 2017-2018 
not meet the screening criteria received free medication according to national guidelines.

\section{Examination of malaria parasites by microscopy and haemoglobin level measurement}

Capillary blood was collected by finger prick and used to prepare thick and thin blood smears in duplicate during study visits. Slides were independently read by two qualified microscopists; results were averaged if they were consistent $(<20 \%$ for the parasite density estimation). If results from the first two readers were discordant, the slide was read by a third qualified microscopist. The final parasitaemia was calculated as the average of the two closest parasitaemia estimations. Only asexual forms were included in the parasite density estimation.

Haemoglobin was measured in capillary blood using a portable haemoglobin analyzer HemoCue $\mathrm{Hb} 201$ System (HemoCue AB, Angelholm, Sweden) on days 0, 14 and 28 or any other days on request of clinicians.

A post-hoc quality control was done on a subset of slides $(n=233)$ at the Noguchi Memorial Institute for Medical Research whereby two microscopists blinded to the original results read the slides. An additional $10 \%$ of the subset were read by a third and fourth microscopist.

\section{Treatment and clinical monitoring during follow-up}

Dispersible 20/120 mg AL tablets $\left(\right.$ Coartem $^{\circledR}$ ) were purchased from LABOREX, a national drug supplier, and DP 20/160 mg and 40/320 mg tablets (D-Artepp ${ }^{\circledR}$ ) were provided by Guilin Pharmaceutical in China. At each study site, a nurse managed the study drugs per the manufacturer's instructions and with recording daily temperature in limited access and air-conditioned storage room. The certificates of analysis of each batch of drugs used on site during the study were requested and archived in the investigator site file.

Each child received a 3-day course of the assigned study drug according to the national guidelines for the treatment of uncomplicated malaria. AL was given in tablets containing $20 \mathrm{mg}$ of artemether and $120 \mathrm{mg}$ of lumefantrine and was given in two daily doses. Children weighing 5-9 kg were given one half of a tablet; children weighing $10-14 \mathrm{~kg}$ were given one tablet, and children weighing $15-24 \mathrm{~kg}$ were given two tablets. DP was given once per day. Children weighing 5-8 $\mathrm{kg}$ and $8-11 \mathrm{~kg}$ were given tablets containing $20 \mathrm{mg}$ of dihydroartemisinin and $160 \mathrm{mg}$ of piperaquine at 1 and 1.5 tablets, respectively. Children weighing 11-17 kg and 17-25 kg were given tablets containing $40 \mathrm{mg}$ of dihydroartemisinin and $320 \mathrm{mg}$ of piperaquine at 1 and 1.5 tablets, respectively. The three once-daily doses for DP and the three twicedaily doses for AL doses were directly supervised by the study team. Doses were not systematically given with food. If a participant vomited within 30 min following drug administration, the full dose was re-administered. In case of persistent vomiting, the child was excluded and treated according to national guidelines for the treatment of severe malaria.

Children were followed for 42 days regardless of drug arm with clinical and laboratory examinations performed on days $0,1,2,3,7,14,21,28,35$ and 42 . Caregivers of study participants were asked to bring their children to the health facility at any time if they felt unwell between scheduled visits. Children who did not attend scheduled visits by mid-day were visited at home by a member of the study team and their caregivers encouraged to bring the child to the health facility for the study procedures. Children were classified as lost to follow-up if they were not seen for each scheduled visit through the end of the study. Children presenting with recurrent parasitaemia were treated per the national guidelines based on clinical presentation [9]. Parents/guardians of participants were provided transportation costs at each scheduled visit.

Drug safety was monitored through questionnaires administered to caregivers and during clinical examination. A severity grading scale, based on the WHO toxicity grading scale was used to assess the severity of reported adverse events and clinical examination findings [24]. Patients with adverse events were assessed, managed in accordance with their clinical status and followed until resolution or stabilization.

All participants were assigned one of the following treatment outcomes according to the $\mathrm{WHO}$ guidelines [7]:

- Early treatment failure: danger signs or severe malaria on days 1-3 with parasitaemia, higher parasitaemia on day 2 than day 0 , parasitaemia on day 3 with a fever or day 3 parasitaemia $\geq 25 \%$ than day 0 ,

- Late clinical failure: danger signs or severe malaria with parasitaemia or parasitaemia with fever between days 4 and last day of follow-up,

- Late parasitological failure: parasitaemia between day 7 and last day of follow-up in absence of a fever,

- Adequate clinical and parasitological response (ACPR): absence of parasitaemia on last day of follow-up, irrespective of fever status, or

- Removed or lost to follow-up.

\section{Sample processing and molecular analysis}

Dried blood spots (DBS) were prepared from 2 to 3 drops of capillary blood spotted on Whatman 903 filter paper on day 0 and any day of recurrence of parasitaemia after day 7. Parasite DNA was extracted using QIAamp DNA blood mini kits (Qiagen, Valencia, CA USA) according 
to the manufacturer's instructions. Plasmodium species was confirmed by PCR on day 0 samples for all treatment failures and a subset of samples for participants classified as ACPR [25]. For recurrent parasitaemias, genotyping using amplification of the merozoite surface proteins 1 and 2 ( $m s p 1$ and $m s p 2$ ), and glutamine-rich protein (glurp) markers was performed on paired samples obtained from participants on day 0 and day of failure.

Primers designed to amplify three allelic families from block two of $m s p 1$ (K1, MAD20, R033), two allelic families from $m s p 2$ (FC27 and IC/3D7), and the polymorphic region of glurp were used in PCR amplification and analysis as previously described [26, 27]. Products from paired samples were loaded adjacent to each other. Gels were stained with ethidium bromide and visualized under UV illumination. Band sizes of PCR products across the three markers were measured visually and compared for paired day 0 and day of failure samples. Band sizes that were equal on day 0 and day of failure were considered to match. If there was at least one matching band in any allelic family for all three markers, the failure was classified as a recrudescence (regardless of whether there were additional or missing alleles). If there were no shared alleles for at least one marker, the failure was classified as a reinfection. If the amplification products failed to result in sharp, defined bands in both day 0 and day of failure samples for a marker, that marker was not used for reinfection and recrudescence determination, but the aforementioned classification criteria were applied for the markers that were amplified.

\section{Markers of anti-malarial drug resistance}

All treatment failures and a random subset of samples from patients classified as ACPR were analysed for markers of anti-malarial drug resistance to explore associations between treatment outcome and presence of mutations. DNA extracts from day 0 were analysed to detect the presence of mutations in the propeller domain of Pfkelch13 (PF3D7_1343700) and the presence of the N86 allele as previously described and compared across participants classified as ACPR, reinfection, and recrudescence $[28,14]$. Sequencing reactions were carried out with a CFX96 Touch BioRad (Marnes-la-Coquette, France). Amplicons were sent to Eurofins Germany for sequencing, and DNA sequences were analysed to identify specific single nucleotide polymorphisms (SNPs) related to anti-malarial resistance. Electropherograms were analysed with CEQ2000 genetic analysis software (Beckman Coulter, Villepinte, France). Parasites with mixed alleles (in which both wild-type and mutant alleles were present) were included in counts for both wild-type and mutant alleles. Plasmepsin-2 (Pfpm2, PF3D7_1408000) and Pfmdr1 (PF3D7_0523000) copy number variation (CNV) were measured by qPCR using a CFX96 Touch (Bio-Rad, France), relative to the single copy of the $\beta$-tubulin gene (used as reference gene), as previously described [16]. Molecular analyses were done at the Malaria Genetics and Resistance Unit, Department of Parasites and Insect Vectors, Institut Pasteur in Paris, France.

\section{Statistical analysis}

Data collected on case report forms were double entered in CSPro 7.0 (US Census Bureau, Washington DC, USA) then imported and checked in Excel (Microsoft, Redmond, USA). Data were analysed with the WHO Excel software template [29], MedCalc version 12 (Mariakerke, Belgium) and R (R Foundation for Statistical Computing, Vienna, Austria). Patients with new infections during the follow-up period and patients with indeterminate PCR genotyping data were excluded from the PCR-corrected per-protocol analysis and censored on day of failure in the Kaplan-Meier estimates. Results were calculated to day 28 for the AL arms and to days 28 and 42 for the DP arms due to the longer half-life of piperaquine. Presence of Pfkelch13 and Pfmdr1 mutations were compared between day 0 samples from those who successfully cleared their initial infection (outcome of ACPR or reinfection) versus day 0 samples from those who developed recrudescent infection using Fisher's exact test. These groups were also used to compare $\mathrm{CNV}$ at different cutoffs $(1.5,2,2.5$, and 3$)$ and as a continuous variable. Pfpm $2 \mathrm{CNV}$ was analysed in the DP arm and Pfmdr CNV was analysed in the AL arm.

\section{Ethical considerations}

The study protocol was approved by the Institutional Ethics Committee of CNRFP and the Health Research Ethics Committee of Burkina Faso and was conducted in accordance with International Conference on Harmonization and Good Clinical Practices. The trial was registered in the Pan African Clinical Trial Registry (PACTR identifier: PACTR201708002499311) and approved by the Regulatory authority from the Ministry of Health. Staff from the Centers for Disease Control and Prevention $(\mathrm{CDC})$ provided technical assistance; the protocol was approved as a non-research program evaluation by the Office of the Associate Director for Science, Center for Global Health at CDC.

\section{Results}

\section{Study profile}

Recruitment took place between November 2017 and September 2018. At day 28 of follow-up, 333 and 339 children in the AL and DP arms, respectively reached study endpoints. At day 42 of follow-up, 337 children 
were evaluated in the DP arm (Table 2, Fig. 2). All groups received the recommended dose of AL or DP except that the dose of AL given to children weighing 5-9 kg was a half tablet instead of 1 full tablet due to an error in the study protocol approved by ethics committees and regulatory authority.

\section{Clinical and parasitological response}

The 672 children reaching endpoints were considered in the efficacy estimates. At day 3, post-treatment parasitaemia was detected in 6 subjects (2\%) in the DP arm and $1(0.2 \%)$ in the AL arm. In the AL arm, there were 177 late treatment failures, of which $54(31 \%)$ were classified as recrudescences, $107(60 \%)$ as reinfections, and $16(9 \%)$ were non-amplified or not available. The uncorrected 28 -day AL per-protocol efficacy was $65 \%(55-74 \%)$ in

Table 2 Baseline characteristics of participants enrolled in the 2017-2018 therapeutic efficacy study, Burkina Faso

\begin{tabular}{|c|c|c|c|c|c|c|}
\hline & \multicolumn{2}{|c|}{ Niangoloko } & \multicolumn{2}{|l|}{ Nanoro } & \multicolumn{2}{|l|}{ Gourcy } \\
\hline & $A L$ & DP & $A L$ & DP & $A L$ & DP \\
\hline Screened & 375 & & 335 & & 324 & \\
\hline Included in analysis (\%) & 116 & 118 & 117 & 113 & 120 & 118 \\
\hline Age in months. mean (SD) & $33.6(13.2)$ & $33.6(13.2)$ & $33.6(13.2)$ & $31.2(12.0)$ & $34.8(12.0)$ & $34.8(12.0)$ \\
\hline Sex (male) n (\%) & $58(50)$ & $68(57.6)$ & $57(48.7)$ & $61(53.0)$ & $59(49.2)$ & $62(52.5)$ \\
\hline Weight (kg). mean (SD) & $12.0(2.7)$ & $12.1(2.7)$ & $11.6(2.0)$ & $11.2(2.2)$ & $11.8(2.2)$ & $11.7(2.3)$ \\
\hline Temperature in ${ }^{\circ} \mathrm{C}$, mean (SD) & $38.3(1.0)$ & $38.3(1.0)$ & $38.2(1.1)$ & $38.1(1.1)$ & $38.1(1.2)$ & $38.2(1.0)$ \\
\hline Parasitaemia day $0^{\mathrm{a}}$ & 36,594 & 36,571 & 48,891 & 42,393 & 42,116 & 33,536 \\
\hline Hemoglobin in g/dl. mean (SD) & $10.2(1.7)$ & $10.0(2.9)$ & $9.9(1.7)$ & $10.1(1.6)$ & $10.0(1.5)$ & $9.9(1.6)$ \\
\hline
\end{tabular}

$A L$ artemether lumefantrine, $D P$ dihydroartemisinin piperaquine, $S D$ standard deviation

a geometric mean parasite density (asexual parasites/ $\mu \mathrm{l}$ )

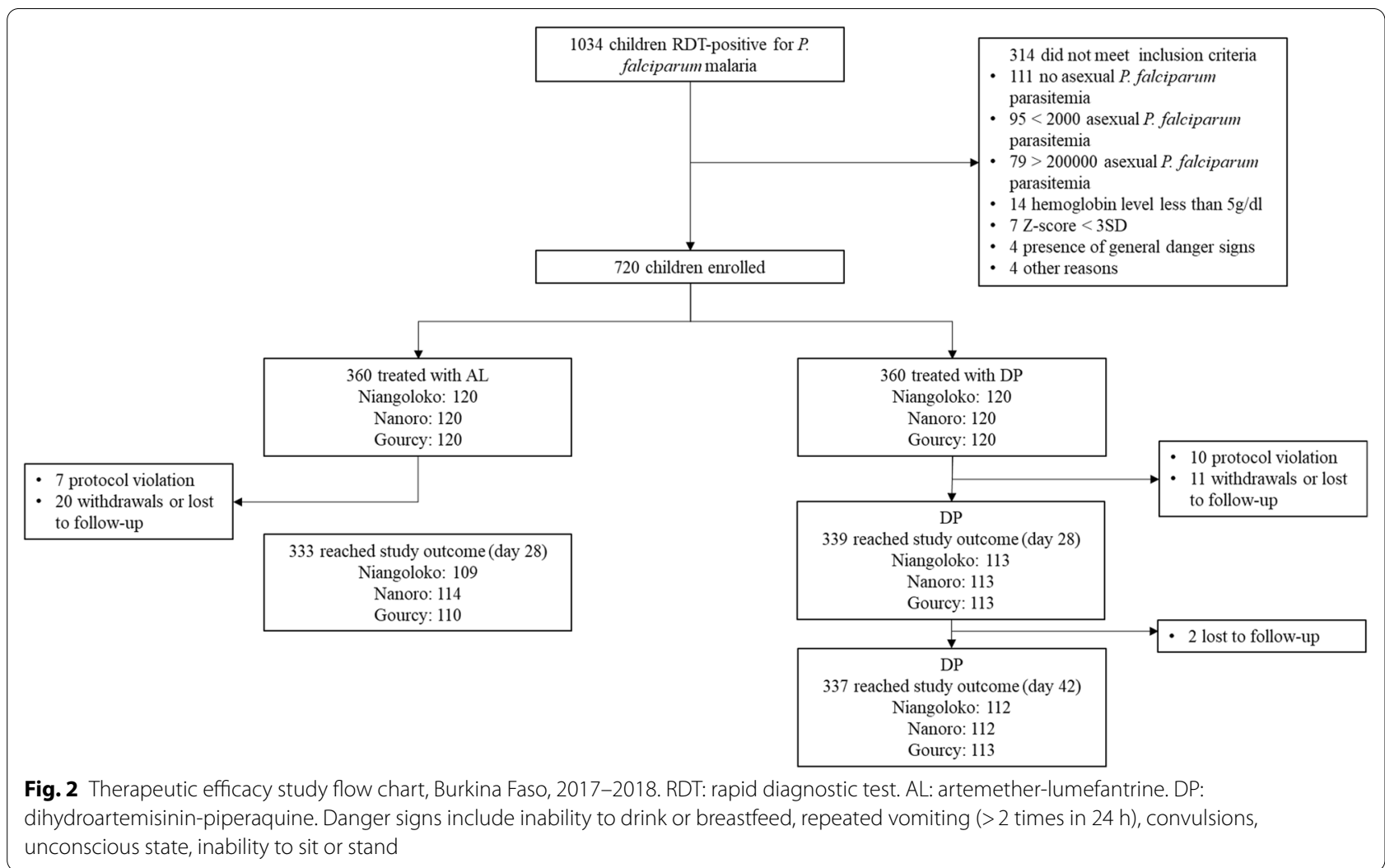


Niangoloko, 27\% (95\% confidence intervals (CI) 19-36\%) in Nanoro, and $48 \%$ (95\% CI 39-58\%) in Gourcy. The PCR-corrected Kaplan-Meier efficacy was 92\% (95\% CI $84-96 \%$ ) in Niangoloko $74 \%$ (95\% CI 64-83\%) in Nanoro, and 76\% (95\% CI 66-83\%) in Gourcy. Excluding the underdosed 5-9 $\mathrm{kg}$ weight group, the PCR uncorrected 28-day AL per-protocol efficacy was 68\% (95\% CI 57-77\%) in Niangoloko, $28 \%$ (95\% CI 20-38\%) in Nanoro, and $44 \%$ (95\% CI 33-55\%) in Gourcy. The PCR-corrected Kaplan-Meier efficacy was 92\% (95\% CI $83-96 \%)$ in Niangoloko, $77 \%$ (95\% CI $65-85 \%)$ in Nanoro, and 73\% (95\% CI 61-81\%) in Gourcy.

In the DP arm, there were 82 late treatment failures at day $42,26(32 \%)$ of which were classified as recrudescences, $48(59 \%)$ as reinfections, and $8(10 \%)$ were non-amplified or not available. The uncorrected 42-day DP per-protocol efficacy was $88 \%$ (95\% CI $80-94 \%$ ) in Niangoloko, 64\% (95\% CI 55-73\%) in Nanoro, and 69\% (95\% CI 60-77\%) in Gourcy. The PCR-corrected 42-day Kaplan-Meier efficacy for DP was 97\% (95\% CI 92-99\%) in Niangoloko, 89 (95\% CI 81-94\%) in Nanoro, and 84\% (95\% CI 75-89\%) in Gourcy (Table 3). Based on genotyping of day 0 samples from late treatment failures, the proportion of infections with a multiplicity of infection $>1$ was estimated at $83 \%(238 / 286)$. The risk of positivity on day 3 was statistically associated with a parasite density $>50,000$ parasites $/ \mu \mathrm{L}$ at day $0(p=0.02$, Chi-squared test). Raw genotyping data are available in the Additional file 1.

Table 3 Uncorrected and PCR-corrected efficacy estimates, Burkina Faso therapeutic efficacy study, 2017-2018

\begin{tabular}{|c|c|c|c|c|c|c|c|c|c|}
\hline & \multicolumn{3}{|l|}{ Niangoloko } & \multicolumn{3}{|l|}{ Nanoro } & \multicolumn{3}{|l|}{ Gourcy } \\
\hline & $A L$ & DP & $\mathrm{DP}$ & $A L$ & DP & $\mathrm{DP}$ & $A L$ & $\mathrm{DP}$ & $\mathrm{DP}$ \\
\hline & 28 Days & 28 Days & 42 Days & 28 Days & 28 Days & 42 Days & 28 Days & 28 Days & 42 Days \\
\hline Enrolled & 120 & 120 & 120 & 120 & 120 & 120 & 120 & 120 & 120 \\
\hline $\begin{array}{l}\text { Reached } \\
\text { study out- } \\
\text { come (\%) }\end{array}$ & 109 (90.8) & $113(94.2)$ & 112 (93.3) & $114(95.0)$ & $113(94.2)$ & $112(93.3)$ & $110(91.7)$ & $113(94.2)$ & $113(94.2)$ \\
\hline $\begin{array}{c}\text { Day } 3 \text { parasi- } \\
\text { taemia }(\%)\end{array}$ & $2(2.0)$ & $0(0.0)$ & $0(0.0)$ & $1(0.9)$ & $2(1.8)$ & $2(1.8)$ & $0(0.0)$ & $4(3.5)$ & $0(0.0)$ \\
\hline $\begin{array}{l}\text { Early treat- } \\
\text { ment failure } \\
(\%)\end{array}$ & $0(0.0)$ & $0(0.0)$ & $0(0.0)$ & $1(0.9)$ & $2(1.8)$ & $2(1.8)$ & $0(0.0)$ & $4(3.5)$ & $4(3.5)$ \\
\hline $\begin{array}{l}\text { Late clinical } \\
\text { failure (\%) }\end{array}$ & 13 (11.9) & $2(1.7)$ & 4 (3.6) & $35(30.7)$ & $2(1.8)$ & $16(14.3)$ & $25(22.7)$ & $2(1.8)$ & $11(9.7)$ \\
\hline $\begin{array}{l}\text { Late parasi- } \\
\text { tological } \\
\text { failure (\%) }\end{array}$ & 25 (22.9) & $1(0.9)$ & $9(8.0)$ & $47(41.2)$ & $6(5.3)$ & 22 (19.6) & $32(29.1)$ & $3(2.7)$ & $20(17.7)$ \\
\hline $\begin{array}{l}\text { Recrudes- } \\
\text { cence }\end{array}$ & 8 & 1 & 3 & 22 & 3 & 9 & 24 & 3 & 14 \\
\hline Reinfection & 23 & 2 & 7 & 55 & 6 & 24 & 29 & 2 & 17 \\
\hline $\begin{array}{l}\text { No PCR result } \\
\text { available }\end{array}$ & 7 & 0 & 3 & 5 & 1 & 5 & 4 & 0 & 0 \\
\hline ACPR & 71 & 111 & 99 & 31 & 103 & 72 & 53 & 104 & 78 \\
\hline $\begin{array}{l}\text { \% ACPR } \\
\text { Uncor- } \\
\text { rected }(95 \% \\
\text { CI) }\end{array}$ & $\begin{array}{l}65.1(55.4- \\
74.0)\end{array}$ & $\begin{array}{l}98.2(93.8- \\
99.8)\end{array}$ & $\begin{array}{l}88,4(80.0- \\
93.7)\end{array}$ & $\begin{array}{c}27.2(19.3- \\
36.3)\end{array}$ & $\begin{array}{l}91.2(84.3- \\
95.7)\end{array}$ & $\begin{array}{l}64.3 \% \\
\quad(54.7-73.1)\end{array}$ & $\begin{array}{l}48.2(38.6- \\
57.9)\end{array}$ & $\begin{array}{l}92.0(85.4- \\
96.3)\end{array}$ & $\begin{array}{l}69.0(59.6- \\
77.4)\end{array}$ \\
\hline $\begin{array}{c}\% \text { ACPR PCR- } \\
\text { Corrected } \\
(95 \% \mathrm{Cl})\end{array}$ & $\begin{array}{l}89.9(81.0- \\
95.5)\end{array}$ & $\begin{array}{l}99.1 \\
\quad(95.1-100)\end{array}$ & $\begin{array}{l}97.1(91.6 \\
99.4\end{array}$ & $\begin{array}{l}57.4(43.2- \\
70.8)\end{array}$ & $\begin{array}{l}97.2(92.0- \\
99.4)\end{array}$ & $\begin{array}{l}86.7 \\
\quad(77.5-93.2\end{array}$ & $\begin{array}{l}68.8(57.3- \\
78.9)\end{array}$ & $\begin{array}{l}93.7(87.4 .- \\
97.4)\end{array}$ & $\begin{array}{l}81.3(72.0- \\
88.5)\end{array}$ \\
\hline \multicolumn{10}{|c|}{ Kaplan-Meier cumulative Efficacy } \\
\hline $\begin{array}{l}\text { Uncorrected } \\
\qquad(95 \% \mathrm{Cl})\end{array}$ & $\begin{array}{l}65.5(55.8- \\
73.6)\end{array}$ & $\begin{array}{l}98.2(93.1- \\
99.6)\end{array}$ & $\begin{array}{l}88.5(88.1- \\
93.2)\end{array}$ & $\begin{array}{l}27.8(19.9- \\
36.2)\end{array}$ & $\begin{array}{l}91.2(84.2- \\
95.1)\end{array}$ & $\begin{array}{l}64.3(54.7- \\
72.4)\end{array}$ & $\begin{array}{c}48.7(39.1- \\
57.6)\end{array}$ & $\begin{array}{l}92.0(85.1- \\
95.7)\end{array}$ & $\begin{array}{l}69.1(59.7- \\
76.7)\end{array}$ \\
\hline $\begin{array}{l}\text { PCR- } \\
\text { corrected } \\
(95 \% \mathrm{Cl})\end{array}$ & $\begin{array}{l}91.6(83.9- \\
95.7)\end{array}$ & $\begin{array}{l}99.1(93.9- \\
99.9)\end{array}$ & $\begin{array}{l}97.2(91.7- \\
99.1)\end{array}$ & $\begin{array}{l}74.4(63.5- \\
82.5)\end{array}$ & $\begin{array}{l}97.3(92.0- \\
99.1)\end{array}$ & $\begin{array}{l}88.7(80.5 \\
93.6)\end{array}$ & $\begin{array}{c}75.6(65.8- \\
83.0)\end{array}$ & $\begin{array}{c}93.9(87.6- \\
97.0)\end{array}$ & $\begin{array}{l}83.6(75.2- \\
89.3)\end{array}$ \\
\hline
\end{tabular}

$A L$ artemether lumefantrine, $D P$ dihydroartemisinin piperaquine, $C l$ confidence intervals, $P C R$ polymerase chain reaction, $A C P R$ adequate clinical and parasitological response 


\section{Safety}

The most frequently reported adverse events during 28 days of follow-up were vomiting (12.4\%), cough (5.4\%) and abdominal pain (2.4\%) (Table 4). No meaningful variation in haemoglobin level was observed in any study arm (Additional file 2: Table S1). One serious adverse event occurred. A 35-month old girl in the Gourcy AL arm died on day 37. She was seen on day 36 for report of fever which had been treated at home with $250 \mathrm{mg}$ of paracetamol on the previous day. A blood smear examination found 15,346 asexual $P$. falciparum parasites/ $\mu$ l. She was immediately treated with oral quinine as per the national treatment guidelines [9]. The caregivers were advised to bring the child back if her condition did not improve. That night, as the condition of the child was deteriorating, she was taken to a traditional healer from whom she received an unknown treatment. The child died at home later the same day.

\section{Assessment of molecular markers associated with antimalarial drug resistance}

A total of 373 day 0 samples were tested for the presence of SNPs; results were interpretable for 367 (98\%). Most parasites $(95.9 \%)$ had a wild-type Pfkelch13 gene. Eight $P$. falciparum isolates $(2.3 \%)$ had synonymous mutations and 7 (1.9\%) had non-synonymous mutations. None of the non-synonymous mutations found have been associated with artemisinin resistance [12]. Of note, Pfkelch13 N629Y $(n=1)$ and V517I $(n=2)$ mutants were observed in day 0 isolates collected from patients treated with $\mathrm{AL}$ and classified as recrudescences (Table 5). No associations between day-3 parasitaemia or treatment outcome and presence of Pfkelch13 mutation was found $(p=1$, $p=0.14$, Fisher's exact test).

To investigate mutations in the Pfmdr1 gene, 362 samples were tested; 318 (88\%) yielded interpretable data.
Most isolates (303/318; 95.3\%) had the N86 allele; 15/318 (4.7\%) carried the $86 \mathrm{Y}$ mutation only; 5 samples had mixed infections consisting of parasites with a wild-type allele and $86 \mathrm{Y}$ mutation. No statistically significant association was found between treatment outcome and presence of the $86 \mathrm{Y}$ mutation (Table 5).

In the DP arm, 131 of 136 (96.3\%) samples were amplified for measurement of Pfpm $2 \mathrm{CNV}$. There was no statistically significant association between treatment failure and Pfpm $2 \mathrm{CNV}$ whether using discrete cut-off points or continuous values. In the AL arm, 235 of 237 (99.2\%) samples were amplified for analysis of Pfmdr1 copy number. No statistical evidence of association between treatment failure and CNV was found for Pfmdr1 (Table 5, Fig. 3).

In the microscopy quality control analysis, the random sample of slides read were concordant, giving a concordance of $88 \%$.

\section{Discussion}

The present study was designed to assess the therapeutic efficacy of AL and DP, two artemisinin-based combinations (ACT) recommended for the treatment of uncomplicated malaria by the NMCP in Burkina Faso among children 6-59 months old. Molecular markers associated with anti-malarial resistance were investigated to provide insight into efficacy results.

In this study, the PCR-corrected ACPR rates at day 28 and 42 were found to be above or equal to $90 \%$ in all the sites for DP at day 28, but below $90 \%$ for Nanoro and Gourcy at day 42, with confidence intervals spanning above $90 \%$ in Gourcy. In the AL arm the 28-day PCRcorrected ACPR rates were below $80 \%$ in two of the three sites (Nanoro and Gourcy), indicating inadequate efficacy of this ACT. The estimated proportion of treatment failure with $\mathrm{AL}(>10 \%)$ contrasts with the low proportion of

Table 4 Adverse events reported among participants enrolled in 2017-2018 therapeutic efficacy study, Burkina Faso

\begin{tabular}{|c|c|c|c|c|c|c|}
\hline & \multicolumn{2}{|l|}{ Niangoloko } & \multicolumn{2}{|l|}{ Nanoro } & \multicolumn{2}{|l|}{ Gourcy } \\
\hline & $A L(n=116)$ & $\mathrm{DP}(n=118)$ & $A L(n=117)$ & $\mathrm{DP}(n=114)$ & $A L(n=120)$ & $D P(n=118)$ \\
\hline Itchiness & 0 & 0 & 0 & 0 & 1 & 0 \\
\hline Otitis media & 1 & 0 & 0 & 0 & 0 & 0 \\
\hline Cough & 14 & 14 & 4 & 1 & 3 & 2 \\
\hline Abdominal pain & 2 & 7 & 2 & 5 & 0 & 1 \\
\hline Skin rash & 0 & 2 & 2 & 0 & 0 & 1 \\
\hline Oral thrush & 0 & 0 & 0 & 0 & 0 & 0 \\
\hline Furunculosis & 0 & 1 & 0 & 0 & 0 & 0 \\
\hline Vomiting & 12 & 23 & 15 & 18 & 6 & 13 \\
\hline Death & 0 & 0 & 0 & 0 & 1 & 0 \\
\hline
\end{tabular}

$A L$ artemether lumefantrine, $D P$ dihydroartemisinin piperaquine 


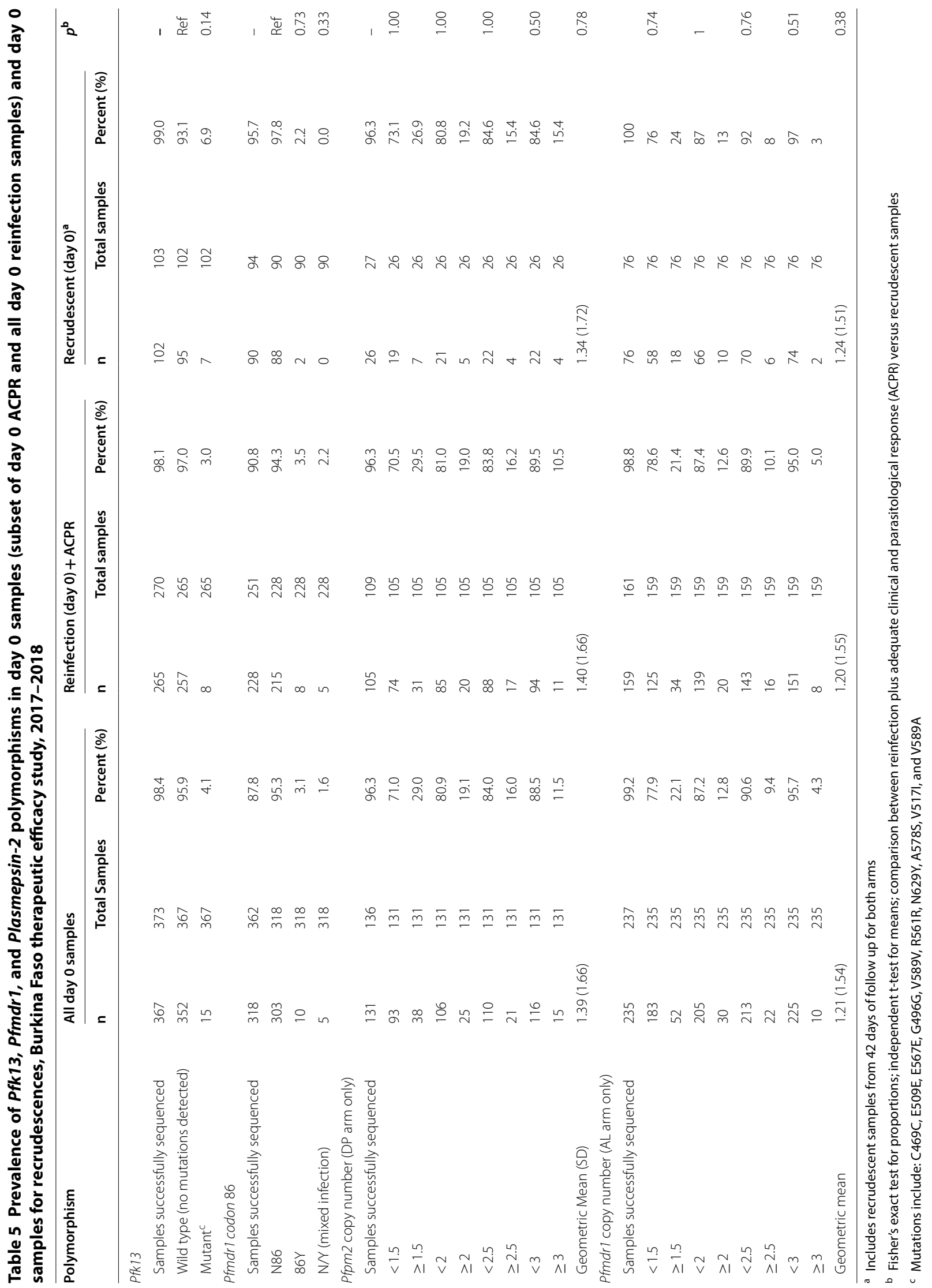



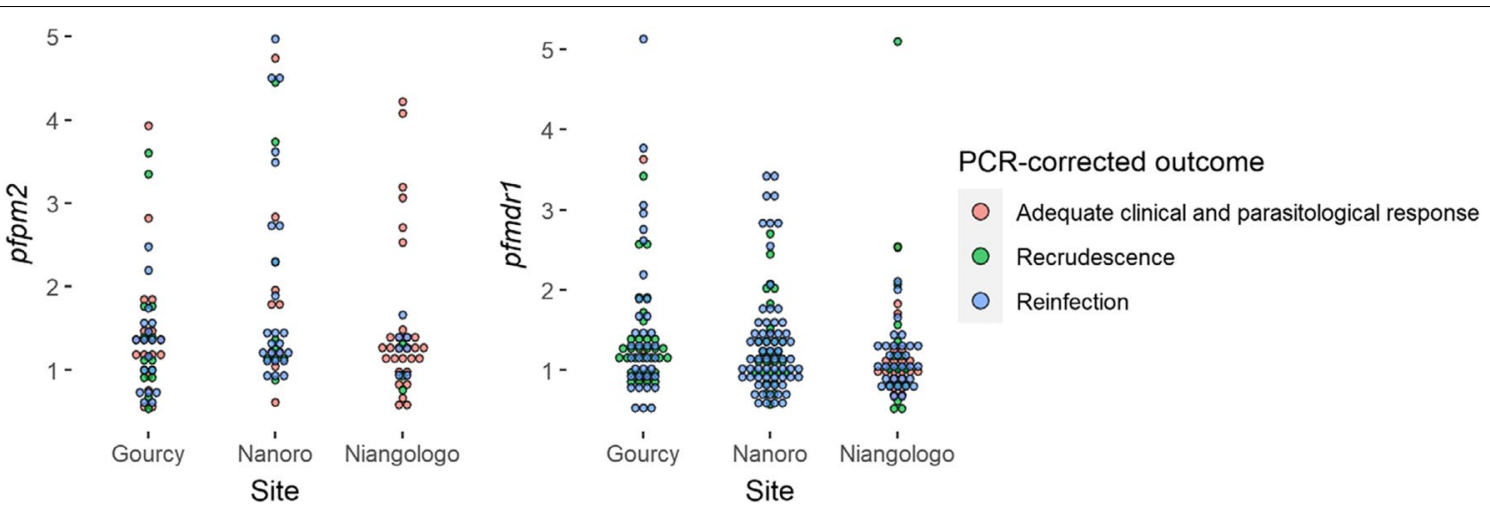

Fig. 3 Copy number variation in dihydroartemisinin-piperaquine arm for pfpm2 copy number $(n=131)$ and artemether-lumefantrine arm for pfmdr $1(n=235)$ in pre-treatment samples collected for therapeutic efficacy monitoring in three sites in Burkina Faso

children still parasitemic at day $3(<2 \%)$ and the absence of Pfkelch13 mutants associated with artemisinin resistance in Day $0 P$. falciparum isolates, suggesting that these $P$. falciparum parasite populations are susceptible to artemisinin derivatives but may have decreased susceptibility to lumefantrine. However, no difference in mutations in the Pfmdr1 N86 allele was found between samples from those who cleared their initial infection versus those who would experience a recrudescent infection. However, there were few participant samples found to carry the $86 \mathrm{Y}$ allele, so there may not have been power to detect a meaningful difference. Furthermore, examining more markers on the Pfmdr1 gene may provide more information about the association between presence of mutations and treatment outcome. The day 3 positivity rates observed in both treatment arms were low $(0-2 \%)$, providing evidence of effective and rapid impact of artemisinin derivatives in reducing the parasite biomass [30].

The PCR-uncorrected treatment failure rates on day 28 observed in the AL arm (ranging from 27 to 65\%) demonstrate a lack of post-treatment prophylactic effect in a setting of high endemicity. This scenario, in which one-third to nearly three-quarters of children return with recurrent parasitaemia after taking $\mathrm{AL}$, has important implications for the health of individuals [31-33], the functioning of the healthcare system, and the need for repeated malaria treatment.

Proportions of treatment failures from previous therapeutic efficacy studies completed in Burkina Faso since 2005 vary from $9.5 \%$ to $23.8 \%$ [20-22]. In these studies, reinfections, ascertained by PCR genotyping, were classified as ACPR (i.e., treatment successes) whereas, according to the WHO guidelines, reinfections should be excluded in the per protocol analysis and censored on the day of reinfection in the Kaplan-Meier analysis [7]. Applying the WHO guidelines to data from these studies, there is evidence that treatment failure rates have been above $10 \%$ for AL in Burkina Faso since 2009, which is more consistent with the current study and shows that the efficacy of AL has been inadequate for several years (Table 1). Continued monitoring in sites that represent the different epidemiologic zones is important to examine heterogeneity of the failure rate by geographic area.

All non-serious adverse events were resolved by the end of the follow-up. Only one serious adverse event occurred in the AL group. The cause of the death was not confirmed but was unlikely associated with the study drug or concomitant medication.

Of six artemisinin-based combinations recommended by the WHO, four are used as first-line treatment of uncomplicated $P$. falciparum malaria in Africa: $\mathrm{AL}$, ASAQ, DP, and artesunate-pyronaridine (AS-PYR) [34]. However, ASAQ is no longer recommended for use in Burkina Faso because SMC with SP-AQ is used nationwide [8], and the present study shows evidence of inadequate 28-day efficacy for $\mathrm{AL}$ and inadequate 42-day efficacy for DP. The last of the four, AS-PYR, has revealed promising results in initial clinical trials in Burkina Faso [35] and warrants further examination in future efficacy studies in the country. However, introducing a new ACT has cost implications for procurement, training of staff, and safety monitoring.

\section{Limitations}

There are several limitations to this study. First, the dose of AL given in the 5-9 $\mathrm{kg}$ weight group, was a half tablet instead of 1 tablet due to an error in the drugs dosing table in the protocol approved by the ethics committees and regulatory authority. However, removing this group from the analysis did not change the efficacy results significantly. Additionally, a fatty food was not given systematically to the children in the AL group 
as recommended by the manufacturer. Some studies have shown that those taking AL on an empty stomach absorb less than $10 \%$ of the lumefantrine in the dose given $[36,37]$. The absence of pharmacokinetic data on lumefantrine did now allow for inference of adequate dosing or absorption of AL. Adding this to future studies may provide insight into associations between $\mathrm{AL}$ absorption and drug efficacy.

Many samples from late treatment failures failed to amplify and were thus excluded in the PCR-corrected per protocol analysis and censored on the day of recurrence in the Kaplan-Meier estimates [7]. This classification method may lead to biased and imprecise efficacy estimates because indeterminate samples are classified the same way as reinfections in the calculation of PCRcorrected efficacy [38]. Among those that did amplify for $m s p 1$ and $m s p 2$, many failed to amplify for glurp, leaving only two markers for determining whether these failures were recrudescences or reinfections. More precise recommended genotyping and analysis methods would help to ameliorate this limitation in future anti-malarial efficacy studies.

Finally, there are limitations in interpretation of $P f p m 2$ and Pfmdr1 CNV in settings where there is high multiplicity of infection due to the inability to quantify the proportion of clones harbouring single or multicopies of Pfpm 2 and Pfmdr1.

\section{Conclusion and recommendations}

The results of this study indicate inadequate efficacy of AL in two sites representing areas with shorter malaria transmission seasons in Burkina Faso, consistent with reanalysis of previous efficacy studies. There is evidence of inadequate efficacy of DP at day 42 in the same two sites. Consideration of a change of first-line ACT may be warranted in Burkina Faso. Further in vivo efficacy studies are planned, which will include dosing of AL according to the manufacturer's instructions, day 7 blood anti-malarial concentration, increased sample size to account for the assumption of a higher failure rate and high rate of reinfection, and inclusion of artesunate-pyronaridine.

\section{Supplementary Information}

The online version contains supplementary material available at https://doi. org/10.1186/s12936-021-03585-6.

Additional file 1. Raw genetic data from day 0 and day of failure samples used to distinuish reinfections from recrudescences among late treatment failures, therapeutic efficacy monitoring, Burkina Faso, 2017-2018.

Additional file 2: Table S1. Hemoglobin g/dl measured among participants in therapeutic efficacy monitoring, 2017-2018, Burkina Faso

\section{Acknowledgements}

To the Noguchi Memorial Institute, Accra, Ghana, health districts of Banfora, Nanoro and Gourcy.

\section{Disclaimer}

The findings and conclusions in this paper are those of the authors and do not necessarily represent the views of the U.S. Centers for Disease Control and Prevention.

\section{Authors' contributions}

AG, EO, CT, ES, MP, HT, IV \& CSC supported study design, data collection, analysis and interpretation of results. DM, IY, \& PS performed laboratory analysis and interpretation of data. OB \& BD provided study oversight and data interpretation. AG, LFM, DK \& ST supported data analysis and manuscript preparation. All authors read and approved the final manuscript.

\section{Funding}

The study was funded by the US President's Malaria Initiative through the Improving Malaria Care Project.

\section{Availability of data and materials}

All data generated or analysed during this study are available from the corresponding author upon reasonable request.

\section{Ethics approval and consent to participate}

The study protocol was approved by the Institutional Ethics Committee of CNRFP and the Health Research Ethics Committee of Burkina Faso and was conducted in accordance with International Conference on Harmonization and Good Clinical Practices. The study was approved by the Regulatory authority from the Ministry of Health. Parents or guardians provided written informed consent for their children to participate in the study.

\section{Competing interests}

All co-authors have no conflicts of interest to declare.

\section{Author details}

${ }^{1}$ Centre National de Recherche Et de Formation Sur Le Paludisme, Ouagadougou, Burkina Faso. ${ }^{2}$ Division of Parasitic Diseases and Malaria, Center for Global Health, Centers for Disease Control and Prevention, US President's Malaria Initiative, Atlanta, GA, USA. ${ }^{3}$ Malaria Genetics and Resistance Unit, Department of Parasites and Insect Vectors, Institut Pasteur, Paris, France. ${ }^{4}$ IRSS / Unité de Recherche Clinique de Nanoro, Nanoro, Burkina Faso. ${ }^{5}$ National Malaria Control Programme, Ouagadougou, Burkina Faso. ${ }^{6} \mathrm{JHPIEGO/Improving} \mathrm{Malaria}$ Care, Ouagadougou, Burkina Faso.

Received: 19 October 2020 Accepted: 7 January 2021

Published online: 19 January 2021

\section{References}

1. World Health Organization. World malaria report 2019. Geneva: World Health Organization; 2019.

2. Diallo A, Sié A, Sirima S, Sylla K, Ndiaye M, Bountogo M, et al. An epidemiological study to assess Plasmodium falciparum parasite prevalence and malaria control measures in Burkina Faso and Senegal. Malar J. 2017;16:63.

3. Ouédraogo AL, Gonçalves BP, Gnémé A, Wenger EA, Guelbeogo MW, Ouédraogo A, et al. Dynamics of the human infectious reservoir for malaria determined by mosquito feeding assays and ultrasensitive malaria diagnosis in Burkina Faso. J Infect Dis. 2016;213:90-9.

4. Bloland PB. Drug resistance in malaria. Geneva: World Health Organization; 2001.

5. Gansané A, Nébié I, Soulama I, Tiono A, Diarra A, Konaté AT, et al. [Change of antimalarial first-line treatment in Burkina Faso in 2005](in French). Bull Soc Path Exot. 2009:102:31-5.

6. Gansané A, Nebié I, Diarra A, Quedraogo A, Soulama I, Bougouma E, et al. Efficacy of chloroquine and sulfadoxine-pyrimethamine for the treatment of uncomplicated malaria in Ouagadougou, capital city of Burkina Faso. Acta Trop. 2005;95:S1. 
7. World Health Organization. Methods for surveillance of antimalarial drug efficacy. Geneva: World Health Organization; 2009.

8. WHO. Policy recommendation: seasonal malaria chemoprevention (SMC) for Plasmodium falciparum malaria control in highly seasonal transmission areas of the Sahel sub-region in Africa. Geneva: World Health Organization; 2012.

9. Programme National de Lutte contre le Paludisme. Directives nationales pour la prise en charge du paludisme dans les formations sanitaires du Burkina Faso. Ouagadougou: Ministère de la Santé; 2017.

10. Menard D, Dondorp A. Antimalarial drug resistance: a threat to malaria elimination. Cold Spring Harbor Perspect Med. 2017;7:a025619.

11. Severini C, Menegon M. Resistance to antimalarial drugs: An endless world war against Plasmodium that we risk losing. J Glob Antimicrob Resist. 2015:3:58-63.

12. World Health Organization. Artemisinin resistance and artemisinin-based combination therapy efficacy: status report. Geneva: World Health Organization; 2018.

13. Uwimana A, Legrand E, Stokes BH, Ndikumana J-LM, Warsame M, Umulisa $\mathrm{N}$, et al. Emergence and clonal expansion of in vitro artemisinin-resistant Plasmodium falciparum kelch13 R561H mutant parasites in Rwanda. Nat Med. 2020;26:1602-8.

14. Venkatesan M, Gadalla NB, Stepniewska K, Dahal P, Nsanzabana C, Moriera C, et al. Polymorphisms in Plasmodium falciparum chloroquine resistance transporter and multidrug resistance 1 genes: parasite risk factors that affect treatment outcomes for $P$. falciparum malaria after artemether-lumefantrine and artesunate-amodiaquine. Am J Trop Med Hyg. 2014;91:833-43.

15. Amato $R$, Lim $P$, Miotto $O$, Amaratunga $C$, Dek D, Pearson RD, et al. Genetic markers associated with dihydroartemisinin-piperaquine failure in Plasmodium falciparum malaria in Cambodia: a genotype-phenotype association study. Lancet Infect Dis. 2017:17:164-73.

16. Witkowski B, Duru V, Khim N, Ross LS, Saintpierre B, Beghain J, et al. A surrogate marker of piperaquine-resistant Plasmodium falciparum malaria: a phenotype-genotype association study. Lancet Infect Dis. 2017:17:174-83.

17. Dama S, Niangaly H, Djimde M, Sagara I, Guindo CO, Zeguime A, et al. A randomized trial of dihydroartemisinin-piperaquine versus artemetherlumefantrine for treatment of uncomplicated Plasmodium falciparum malaria in Mali. Malar J. 2018;17:347.

18. Koita OA, Sangaré L, Miller HD, Sissako A, Coulibaly M, Thompson TA, et al, AQ-13, an investigational antimalarial, versus artemether plus lumefantrine for the treatment of uncomplicated Plasmodium falciparum malaria: a randomised, phase 2, non-inferiority clinical trial. Lancet Infect Dis. 2017;17:1266-75

19. Denoeud-Ndam L, Dicko A, Baudin E, Guindo O, Grandesso F, Diawara H, et al. Efficacy of artemether-lumefantrine in relation to drug exposure in children with and without severe acute malnutrition: an open comparative intervention study in Mali and Niger. BMC Med. 2016;14:167.

20. Siribié M, Diarra A, Tiono A, Soulama I, Sirima S. Efficacité de l'artémétherluméfantrine dans le traitement du paludisme simple de l'enfant en milieu rural au Burkina Faso en 2009. Bull Soc Path Exot. 2012;105:202-7.

21. Sondo P, Derra K, Diallo-Nakanabo S, Tarnagda Z, Zampa O, Kazienga $A$, et al. Effectiveness and safety of artemether-lumefantrine versus artesunate-amodiaquine for unsupervised treatment of uncomplicated falciparum malaria in patients of all age groups in Nanoro, Burkina Faso: a randomized open label trial. Malar J. 2015;14:325.
22. Tinto H, Diallo S, Zongo I, Guiraud I, Valea I, Kazienga A, et al. Effectiveness of artesunate-amodiaquine vs. artemether-lumefantrine for the treatment of uncomplicated falciparum malaria in Nanoro, Burkina Faso: a non-inferiority randomised trial. Trop Med Int Health. 2014;19:469-75.

23. DGESS. Annuaire statistique Ouagadougou. Burkina Faso: Ministère de la Santé; 2018. p. 2018.

24. WHO. Toxicity grading scale for determining the severity of adverse events. Geneva: World Health Organization; 2003.

25. Canier L, Khim N, Kim S, Sluydts V, Heng S, Dourng D, et al. An innovative tool for moving malaria PCR detection of parasite reservoir into the field. Malar J. 2013;12:405.

26. Snounou G, Beck H. The use of PCR genotyping in the assessment of recrudescence or reinfection after antimalarial drug treatment. Parasitol Tdy. 1998; 14:462-7.

27. $\mathrm{WHO}, \mathrm{MMV}$. Methods and techniques for clinical trials on antimalarial drug efficacy: Genotyping to identify parasite populations. Geneva, World Health Organization, 2008. https://www.who.int/malaria/publications/ atoz/9789241596305/en/

28. Ménard D, Khim N, Beghain J, Adegnika AA, Shafiul-Alam M, Amodu O, et al. A worldwide map of Plasmodium falciparum K13-propeller polymorphisms. N Engl J Med. 2016;374:2453-64.

29. WHO. Tools for monitoring antimalarial drug efficacy. Geneva, World Health Organization, 2019. http://www.who.int/malaria/areas/drug_resis tance/efficacy-monitoring-tools/en/. Accessed 13 Jun 2020.

30. White N. Antimalarial drug resistance and combination chemotherapy. Philos Trans R Soc Lond B Biol Sci. 1999;354:739-49.

31. Fernando SD, Rodrigo C, Rajapakse S. The, "hidden" burden of malaria: cognitive impairment following infection. Malar J. 2010;9:366.

32. Foote EM, Sullivan KM, Ruth LJ, Oremo J, Sadumah I, Williams TN, et al. Determinants of anemia among preschool children in rural, Western Kenya. Am J Trop Med Hyg. 2013;88:757-64.

33. McCuskee S, Brickley EB, Wood A, Mossialos E. Malaria and macronutrient deficiency as correlates of anemia in young children: a systematic review of observational studies. Ann Glob Health. 2014;80:458-65.

34. WHO Prequalification of Medicines Programme. Geneva: World Health Organization; 2020. Accessed $20 \mathrm{Jul} 2020$.

35. Sagara I, Beavogui AH, Zongo I, Soulama I, Borghini-Fuhrer I, Fofana B, et al. Safety and efficacy of re-treatments with pyronaridine-artesunate in African patients with malaria: a substudy of the WANECAM randomised trial. Lancet Infect Dis. 2016:16:189-98.

36. COARTEM ${ }^{\circledR}$ (artemether and lumefantrine) tablets, for oral use. Novartis Pharmaceuticals Corporation; 2019

37. White NJ, van Vugt M, Ezzet FD. Clinical pharmacokinetics and pharmacodynamics of artemether-lumefantrine. Clin Pharmacokinet. 1999:37:105-25.

38. Dahal P, Stepniewska K, Guerin PJ, D'Alessandro U, Price RN, Simpson JA. Dealing with indeterminate outcomes in antimalarial drug efficacy trials: a comparison between complete case analysis, multiple imputation and inverse probability weighting. BMC Med Res Methodol. 2019;19:215.

\section{Publisher's Note}

Springer Nature remains neutral with regard to jurisdictional claims in published maps and institutional affiliations.

Ready to submit your research? Choose BMC and benefit from:

- fast, convenient online submission

- thorough peer review by experienced researchers in your field

- rapid publication on acceptance

- support for research data, including large and complex data types

- gold Open Access which fosters wider collaboration and increased citations

- maximum visibility for your research: over 100M website views per year

At BMC, research is always in progress.

Learn more biomedcentral.com/submissions 